\title{
Recurrent volvulus of the transverse colon after sigmoid resection
}

\author{
K. A. C. Booij • P. J. Tanis • T. M. van Gulik • \\ D. J. Gouma
}

Accepted: 29 July 2008 / Published online: 15 August 2008

(C) The Author(s) 2008. This article is published with open access at Springerlink.com

\section{Dear Editor:}

Volvulus of the large bowel is a relatively rare cause of colonic obstruction in Western countries, with an incidence of up to $6 \%$. Volvulus of the colon is mostly located in the sigmoid (43-80\%) and caecum (15-43\%). Rarely, a volvulus of the transverse colon or splenic flexure occurs due to fixation of this part of the colon by the phrenicocolic, gastrocolic and splenocolic ligaments.

Risk factors for developing large bowel volvulus are congenital anatomical variations such as increased colonic length and abnormal fixation, Chagas disease resulting in megacolon, chronic constipation, distal obstruction such as carcinoma of the sigmoid, previous abdominal surgery and pregnancy. There is a male predominance and a geographical variation, with a higher incidence in African, Asian and Eastern countries.

A 39-year-old, mentally disabled man was presented to the emergency department with progressive abdominal distension and pain since day 1 . He complained of nausea, vomiting and fever of up to $38.5^{\circ} \mathrm{C}$. Time of last bowel movement was unclear. He had a medical history of chronic constipation, epilepsy and hydrocephalus with placement of two ventriculoperitoneal (VP) shunts at a young age. These drains needed revision because of dysfunction a few times thereafter. The patient lives in a sheltered home.

During physical examination, a moderately ill man was seen, with an evident enlargement of the skull (circumference $73 \mathrm{~cm}$ ). His consciousness was clear and there were no signs of meningitis. The abdomen was distended with high-

K. A. C. Booij $(\bowtie) \cdot$ P. J. Tanis · T. M. van Gulik • D. J. Gouma Department of Surgery, Academic Medical Center,

Meibergdreef 9 ,

1105 AZ Amsterdam, the Netherlands

e-mail: K.A.Booij@amc.uva.nl pitched bowel sounds and diffuse pain but without signs of peritonitis. Blood analysis showed a C-reactive protein (CRP) of $73 \mathrm{mg} / \mathrm{L}$ and a white blood cell count of $11 \times 10^{9}$ per litre. An abdominal X-ray showed a large bowel obstruction and coprostasis. Conservative management during $24 \mathrm{~h}$, consisting of nasogastric tube drainage and enemas, did not result in relief of complaints. A laparotomy was performed, where a significantly distended sigmoid colon was seen (diameter $15 \mathrm{~cm}$ ) because of rotation around its own mesentery. This had resulted in distension of the proximal part of the colon and small bowel. Adhesiolysis was performed, followed by resection of the sigmoid and reconstruction by end-to-end anastomosis. On day 4 postoperatively, the patient was discharged without any complications.

Four years later, at the age of 43 years, the patient was presented again to the emergency department with progressive abdominal pain, nausea and vomiting over a period of several days. His last bowel movement had been a few days ago, despite the use of enemas. At clinical examination, the patient was moderately ill with a temperature of $39^{\circ} \mathrm{C}$. There were no signs of meningitis. The abdomen was very much distended, with high-pitched bowel sounds and diffusely localized pain during palpation. Because of the stressed abdominal wall, signs of peritoneal reaction could not be adequately tested. Laboratory testing revealed a CRP of $216 \mathrm{mg} / \mathrm{L}$ and a white blood cell count of $20 \times 10^{9}$ per litre. On abdominal X-ray, maximum diameter of the colon was $13.5 \mathrm{~cm}$. With a diagnosis of recurrent bowel obstruction with signs of infection and a substantial risk of blowout, an emergency laparotomy was performed. At laparotomy, a volvulus of the transverse colon was found. The bowel was intact, without any signs of ischaemia. The VP shunts were identified after adhesiolysis. Due to the history of chronic constipation and the recurrence of volvulus, a subtotal colectomy with side-to-end ileorectal anastomosis was performed. 
Postoperatively, fever and elevated parameters of infection persisted, despite intravenously administered antibiotics. A computed tomography (CT) scan of the abdomen on the third postoperative day showed intraperitoneal fluid without extravasation of oral contrast. At relaparotomy, there was no leakage or ischaemia of the anastomosis. Partial resection of omentum was performed because of necrosis. The postoperative course after relaparotomy was uneventful and the patient was discharged 9 days from primary intervention.

The pathogenesis of large bowel volvulus is not completely understood yet. A long redundant sigmoid which easily twists around its often narrow mesenteric attachment to the posterior abdominal wall has probably an embryological origin but is also associated with a high-roughage diet. Another common feature in patients with large bowel volvulus is distension of the colon caused by chronic constipation. This is probably the underlying condition and explanation for the fact that institutionalisation and a medical history involving a psychiatric or neurologic disease have been identified as risk factors for volvulus of the colon.

The presented case completely fits within this risk profile. It is noteworthy that the colon was diffusely dilated at primary laparotomy and that a tension-free anastomosis could only be ensured by mobilisation of the splenic flexure. The presence of a megacolon during primary intervention is a well-known risk factor for recurrence of volvulus and dividing the attachments of the remaining colon theoretically further increases this risk. In the literature, the incidence of recurrent volvulus after previous resection and primary anastomosis is $22 \%$ to $36 \%$. Therefore, it is recommended to consider a subtotal colectomy in the presence of a megacolon, instead of partial resection of the involved bowel segment. In retrospect, there were probably enough arguments to perform a subtotal colectomy at initial presentation of our patient instead of a sigmoid resection.

A few case reports describe the occurrence of a volvulus in the presence of a VP shunt. The small bowel was mostly affected in these patients. The incidence of abdominal complications in the presence of VP shunts is $10 \%$ to $30 \%$. Volvulus can occur in case the VP shunt functions as a leading point for torsion. However, this is mostly reported in children. In the described patient, it is questionable whether the VP shunts have been a causative factor because these shunts were already present for years and turned out to be almost completely covered and fixed by omentum and adhesions.
The diagnosis of volvulus can be made on plain abdominal X-ray in the majority of patients; sensitivity is $65 \%$ for volvulus of the sigmoid. Endoscopy has a detection rate of $100 \%$ and enables assessment of vitality of the bowel. If there are no signs of ischaemia, immediate desufflation can be performed. The investigation of preference, however, is CT scan or magnetic resonance imaging. Using these non-invasive modalities, the diagnosis as well as the location of the volvulus can be established at an early stage with a high accuracy. We omitted these imaging modalities in the present case since the threat of a colonic blowout, fever and elevated infectious parameters were considered to necessitate urgent laparotomy.

The treatment of volvulus depends on its clinical presentation and localisation. Endoscopic desufflation with placement of a rectal cannula in case of a volvulus of the sigmoid is a relatively simple and fast procedure in the absence of ischaemia. Reported success rates in the emergency setting are $85 \%$ to $95 \%$. With advanced age, absence of ischaemia and a high operative risk due to comorbidity, this seems to be the treatment of choice. Elective resection can be considered after successful acute desufflation because of the high recurrence rate of $60 \%$ to $90 \%$. Emergency laparotomy should be performed in the presence of ischaemia, perforation or unsuccessful endoscopic desufflation.

Level 1 evidence for the surgical treatment of large bowel volvulus is scarce. The only randomised controlled trial in this field concluded that primary resection was more successful in comparison with mesosigmoidopexy for patients with sigmoid volvulus and a vital bowel (90\% versus $71.5 \%)$. For patients with signs of ischaemia, resection with primary anastomosis was less successful (50\% versus $87.5 \%$ ) and was associated with a two times higher mortality rate $(33.3 \%$ versus $12.5 \%$ ) compared to resection with colostomy. Colopexy has also been described as a treatment option for volvulus of the transverse colon. However, resection seems to be the treatment of choice in these patients too.

The presented case illustrates that, in the presence of risk factors for volvulus and a megacolon during laparotomy, a subtotal colectomy can be recommended.

Open Access This article is distributed under the terms of the Creative Commons Attribution Noncommercial License which permits any noncommercial use, distribution, and reproduction in any medium, provided the original author(s) and source are credited. 